\title{
An Intelligence System for Detection of Cancer and Diagnosis
}

\author{
Abhishek Kumar Gupta ${ }^{1}$, Kulvinder Singh Mann ${ }^{2}$ \\ ${ }^{I}$ Dept. of CSE, RBCEBTW, Mohali, Student Under PTU \\ ${ }^{2}$ Dept. of IT, GNDEC, Ludhiana
}

\begin{abstract}
Currently the digital images are used in various areas like medical, fashion, architecture, face recognition, finger print recognition and bio metrics. Recently the CBIR reduced the semantic gap between the low visual features and high level image semantics. In this paper we describe a novel framework for performing content-based image retrieval using Back Propagation Neural Network. Our method focuses on performing category search, though it could easily be ex-tended to other types of searches, and does not require relevance feedback in order to perform reasonably. It also emphasizes the importance of utilizing information collected from the sets of image database in medical system.
\end{abstract}

Keywords: Artificial Intelligence, ANN, Back Propagation,CBIR,IRS

\section{Introduction}

The Intelligence System for Detection of Cancer and Diagnosis is based on the concept of artificial intelligence. Warren McCulloch, a neurophysiologist, and a young mathematician, Walter Pitts, explained the concept of Artificial Intelligence in 1943. They designed a model of simple neural network with electrical circuits. Neural networks, with their remarkable ability to derive meaning from complicated or imprecise data, can be used to extract patterns and detect trends that are too complex to be noticed by either humans or other computer techniques. A trained neural network can be thought of as an "expert" in the category of information it has been given to analyze [1]. In general, ANN techniques are a family of mathematical models that are based on the human brain functioning. All ANN methodologies share the concept of "neurons" (also called "hidden units") in their architecture. Each neuron represents a synapse as its biological counterpart. Therefore, each hidden unity is constituted of activation functions that control the propagation of neuron signal to the next layer (e.g. positive weights simulate the excitatory stimulus and negative weights simulate the inhibitory ones). A hidden unit is composed by a regression equation that processes the input information into a non-linear output data. Therefore, if more than one neuron is used to compose an ANN, non-linear correlations can be treated [2].
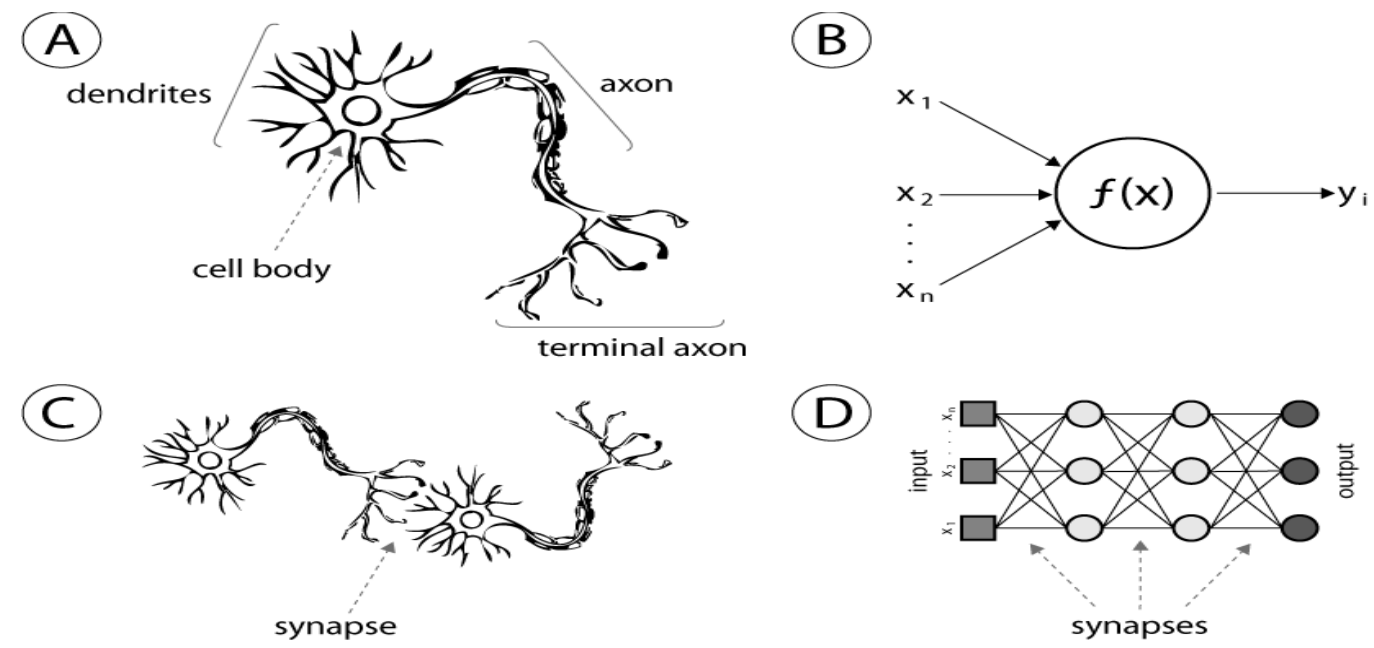

Fig. 1. (A) Human neuron; (B) artificial neuron or hidden unity; (C) biological synapse; (D) ANN synapses

1. Advantages Of Artificial Intelligence Are:

1.1 Adaptive Learning: Adaptive learning is an educational method which is used by the computers as interactive learning techniques. Computers adapt the presentation of educational material according to student's learning needs, as indicated by their response to questions and tasks [3].

1.2 Self-Organisation: An Artificial Neural Network creates its own organization or representation of their information it receives during learning time. 
1.3 Real-Time Operation : ANN computations may be carried out in parallel, and special hardware devices are being designed and manufactured which take advantage of this capability[1].

1.4 Fault tolerance via redundant information coding: Partial destruction of a network leads to the corresponding degradation of performance. However, some network capabilities may be retained even with major network damage.

\section{2. content based image retrival:}

The Content-Base Image Retrieval (CBIR), is also known as Query By Image Content (QBIC) or Content Based Visual Information Retrieval (CBVIR). It is the application of computer vision techniques to the image retrieval problem.

\section{Image Retrieval System (Irs)}

Some years ago, medical information systems only provided textual information about patients in treatment; later, this data is stored in large databases where queries could be made by searching for the text information [5]. Content-based image retrieval (CBIR) technology has been proposed to benefit not only the management of increasingly large image collections, but also to aid clinical care, biomedical research [6]. Our system (System for Detection of Cancer and Diagnosis) display the disease as well as medicine related to particular disease. Fig. 3 describes the process of Image Retrieval System. The image retrieval process consists of two main phases: pre-processing phase and retrieval phase. Both phases are described as follows [7].

\section{Pre-Processing Phase}

Image segmentation is the process of separating or grouping an image into different parts. The goal of image segmentation is to cluster pixels into salient image regions, where the regions corresponding to individual surfaces, objects, or natural parts of objects Image segmentation methods can be divided into three types: Boundary based techniques, Region based techniques, and Pixel based direct classification methods [8].

The pre-processing phase is composed of two main components:

1.1 The Feature Extraction model

1.2 Classification model

The idea behind feature extraction is to use the lowest level pixel values to extract slightly higher level features, such as gray-levels, texture or shape and combine them into an ideally high level representation of the image content [9].

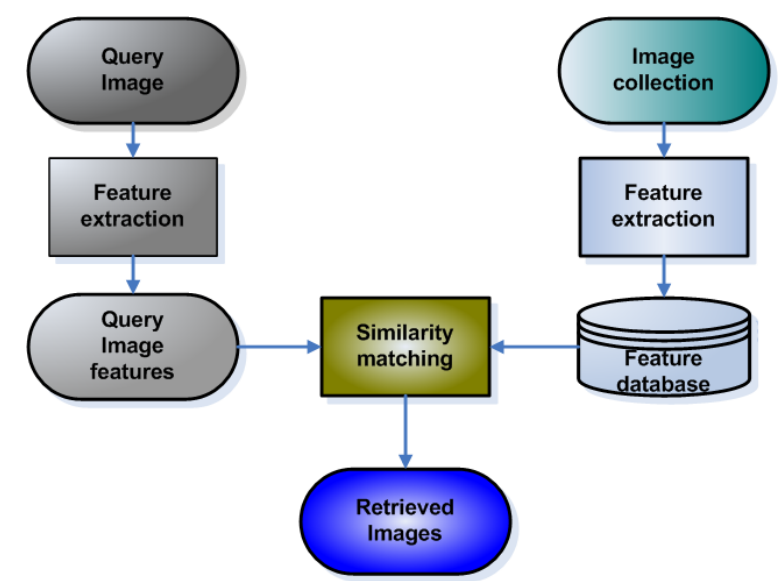

Fig. 2 CBIR System

\section{Retrieval Phase}

This problem is related to the searching for digital images in large databases. The Content-Based Image Retrieval system analyzes the content of image rather then the metadata such as keywords, tags or descriptions associated with the image. In the Content Based Image Retrieval system the term content refers to color, shapes, textures or any other information that can be derived from the image. 

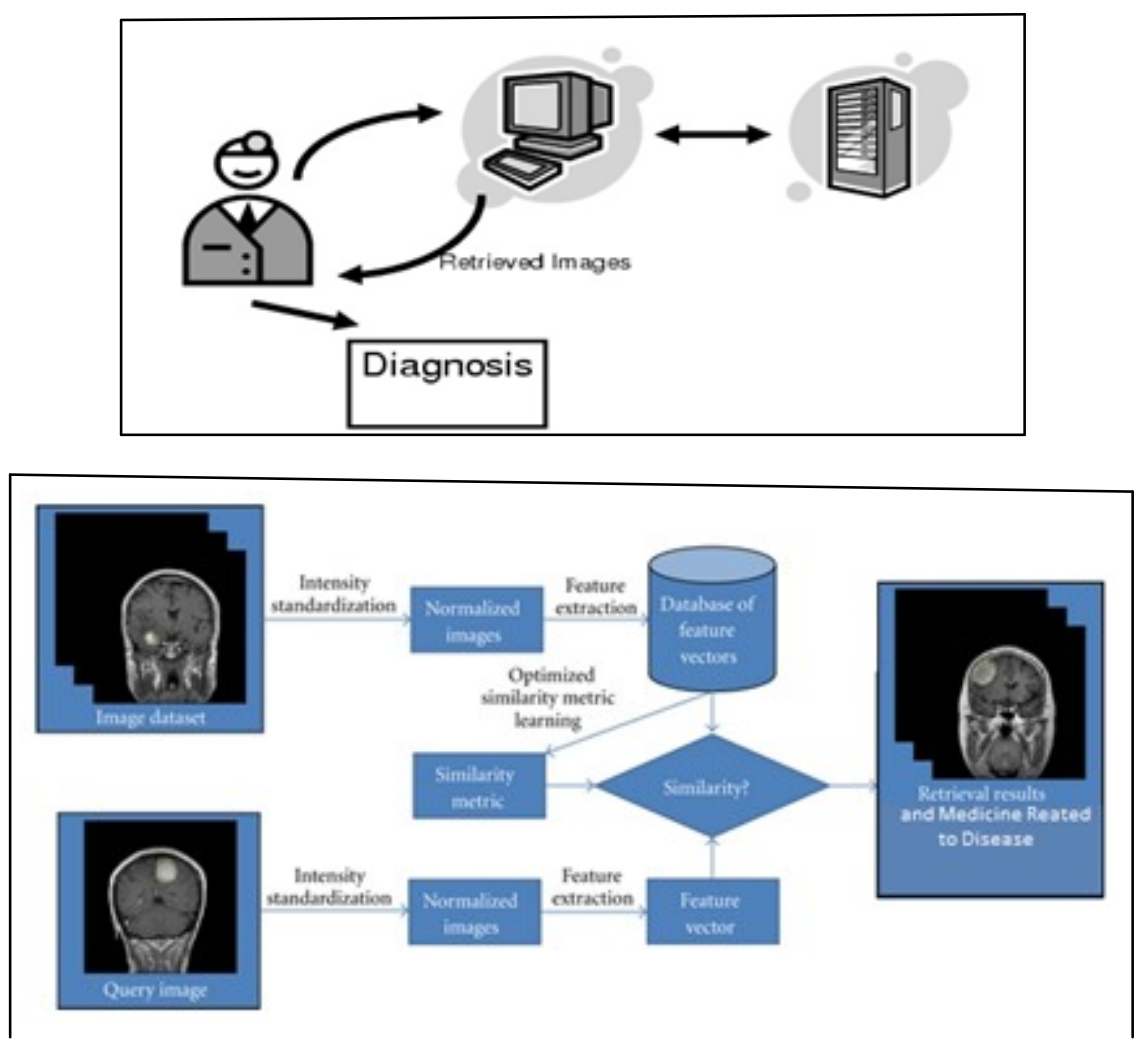

Fig. 3 Cancer Detection System

\section{Proposed System}

We have developed a CBIR system based on feed forward back propagation algorithm techniques for future extraction and similarity measurements. It has been observed from the experimental that feed-forward back propagation neural network gives better results in terms of efficiency, precision and recall. Fig. 4 shows the admin panel. From the admin panel admin become able to update database, register the new patient and we can also see the old history of previous patient.

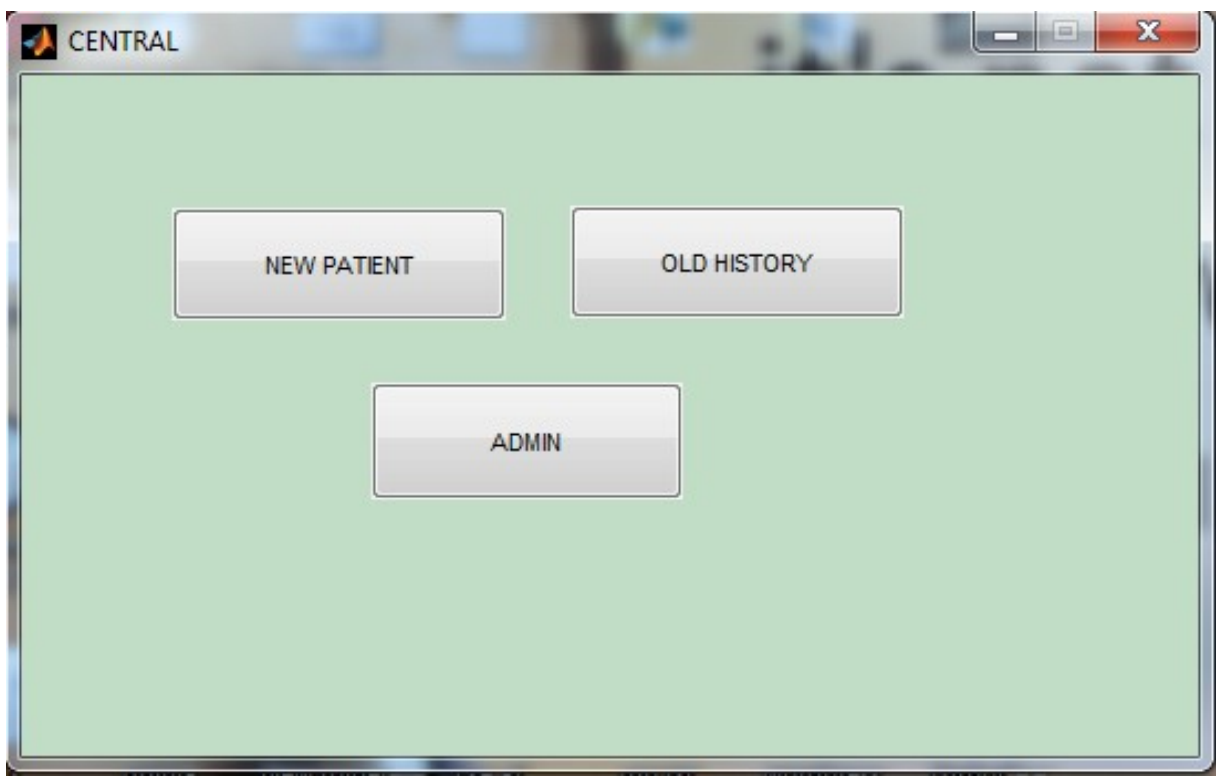

Fig. 4 Admin Panel

Fig. 5 displays the new patient registration form in this window we become able to upload the patient image and information about the patient. When we click the diagnose button it compares the uploaded image 
with the image database. And generates the results see Fig. 6. It will also display the various medicine related to the particular disease. This is the very attractive feature of the system which is implemented by us. And this feature is the very beneficial for the doctor.

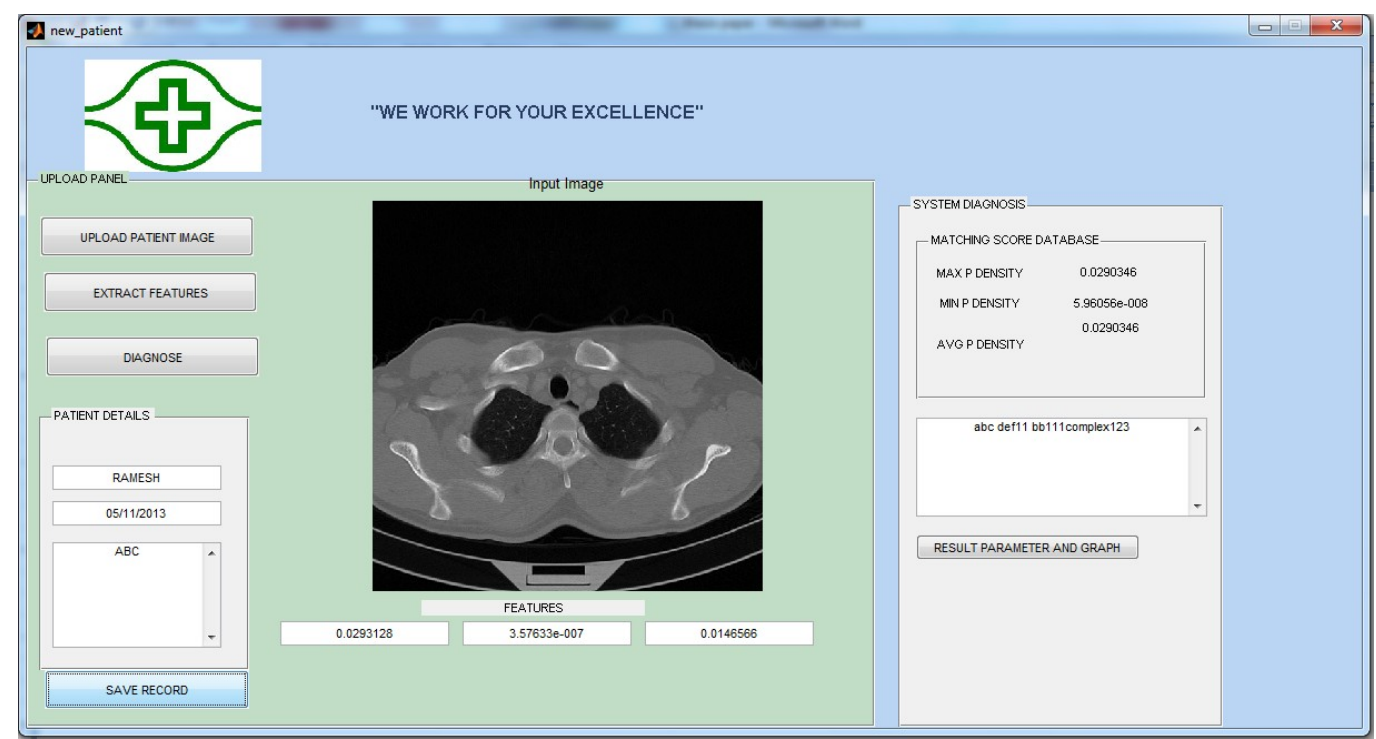

Fig. 5

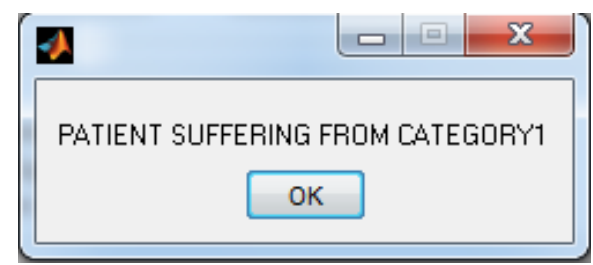

Fig. 6

Fig. 7 displays the old history window for checking the previous records of the patient. From the old history window doctor can check the previous record of the patient.

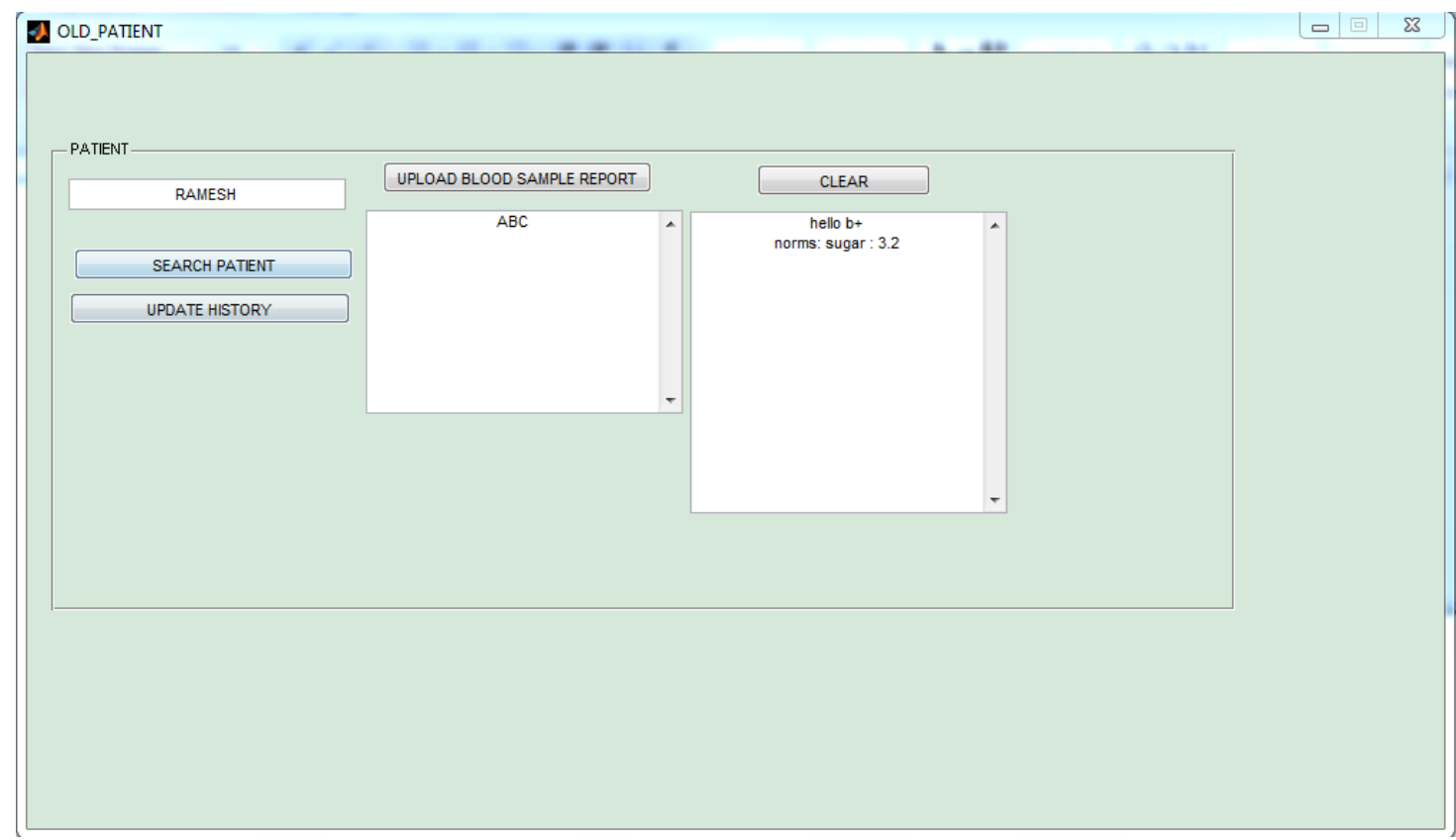

Fig. 7 
Fig. 8 displays the flowchart of our proposed system (An Intelligence system for Detection of Cancer and Diagnosis).

In the flowchart the training process include creation, configuring a three layered neural network and making it learn about the extracted color, texture and edge features of training set images. Training set includes all the images from image database considered. The learning process is carried out using Back Propagation Algorithm, which include computing errors.

In the flowchart the testing phase includes the querying and retrieving task. The query image is first preprocessed and also its features are extracted.

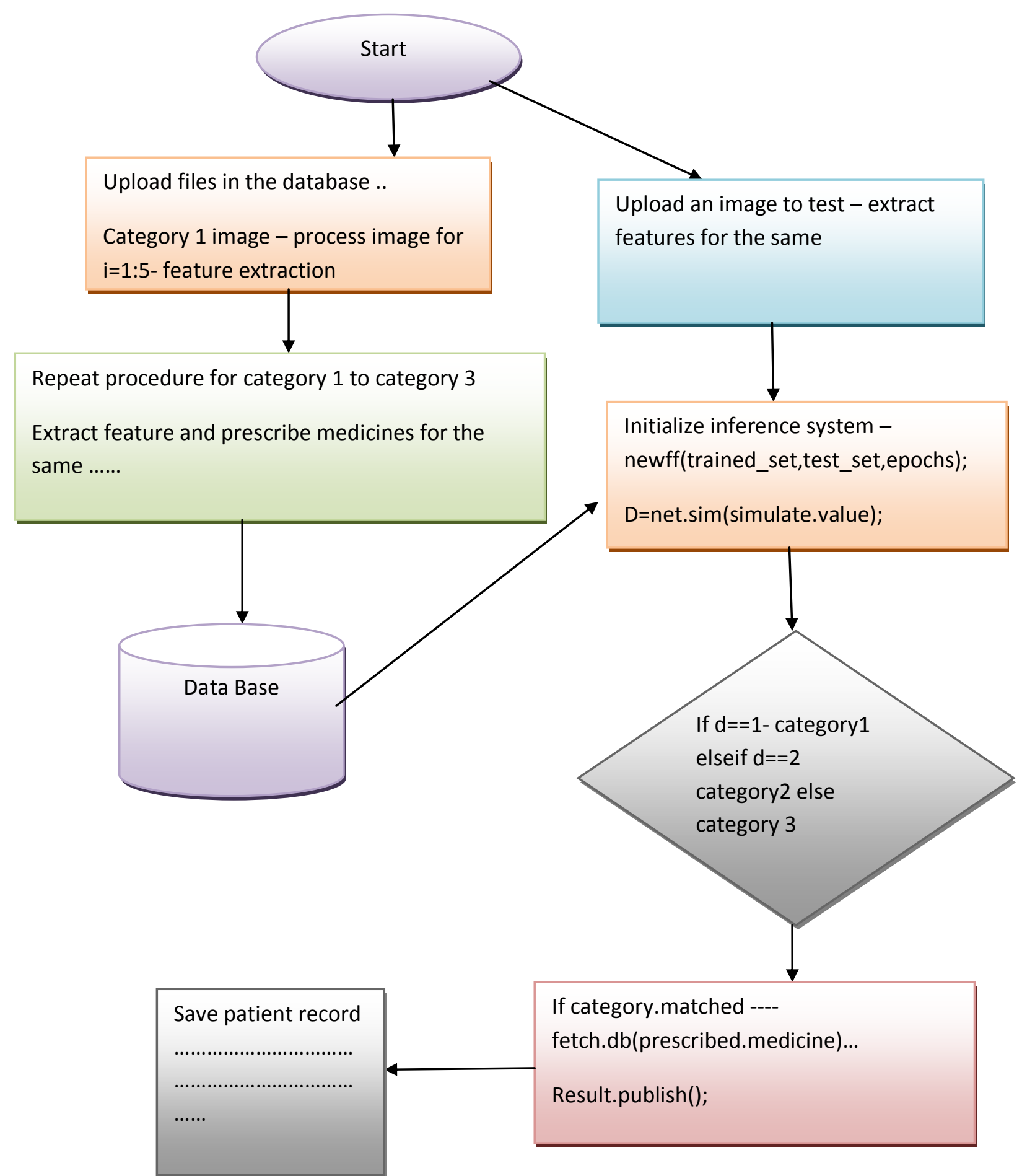

Fig. 8 


\section{Machine Learning Techniques}

We have used the feed forward back propagation Algorithm in our system. This algorithm can be divided into two phases.

\section{Phase 1: Propagation}

Each propagation involves the following steps:

1. Forward propagation of a training pattern's input through the neural network in order to generate the propagation's output activations [10].

2. Backward propagation of the propagation's output activations through the neural network using the training pattern target in order to generate the deltas of all output and hidden neurons [10].

\section{Phase 2: Weight update}

For each weight-synapse follow the following steps:

1. Multiply its output delta and input activation to get the gradient of the weight [10].

2. Subtract a ratio (percentage) of the gradient from the weight [10].

This ratio (percentage) influences the speed and quality of learning; it is called the learning rate. The greater the ratio, the faster the neuron trains; the lower the ratio, the more accurate the training is. The sign of the gradient of a weight indicates where the error is increasing; this is why the weight must be updated in the opposite direction [10].

Repeat phase 1 and 2 until the performance of the network is satisfactory.

\section{Algorithm}

Algorithm for a 3-layer network (only one hidden layer):

Initialize network weights (often small random values)

do for Each training example ex

prediction $=$ neural-net-output $($ network, ex $) / /$ forward pass

actual $=$ teacher-output $(\mathrm{ex})$

compute error (prediction - actual) at the output units

compute for all weights from hidden layer to output layer // backward pass

compute for all weights from input layer to hidden layer // backward pass continued

update network weights

until all examples classified correctly or another stopping criterion satisfied

return the network

\begin{tabular}{l} 
V. Results \\
1.1 Image Database \\
\hline Image 1
\end{tabular}




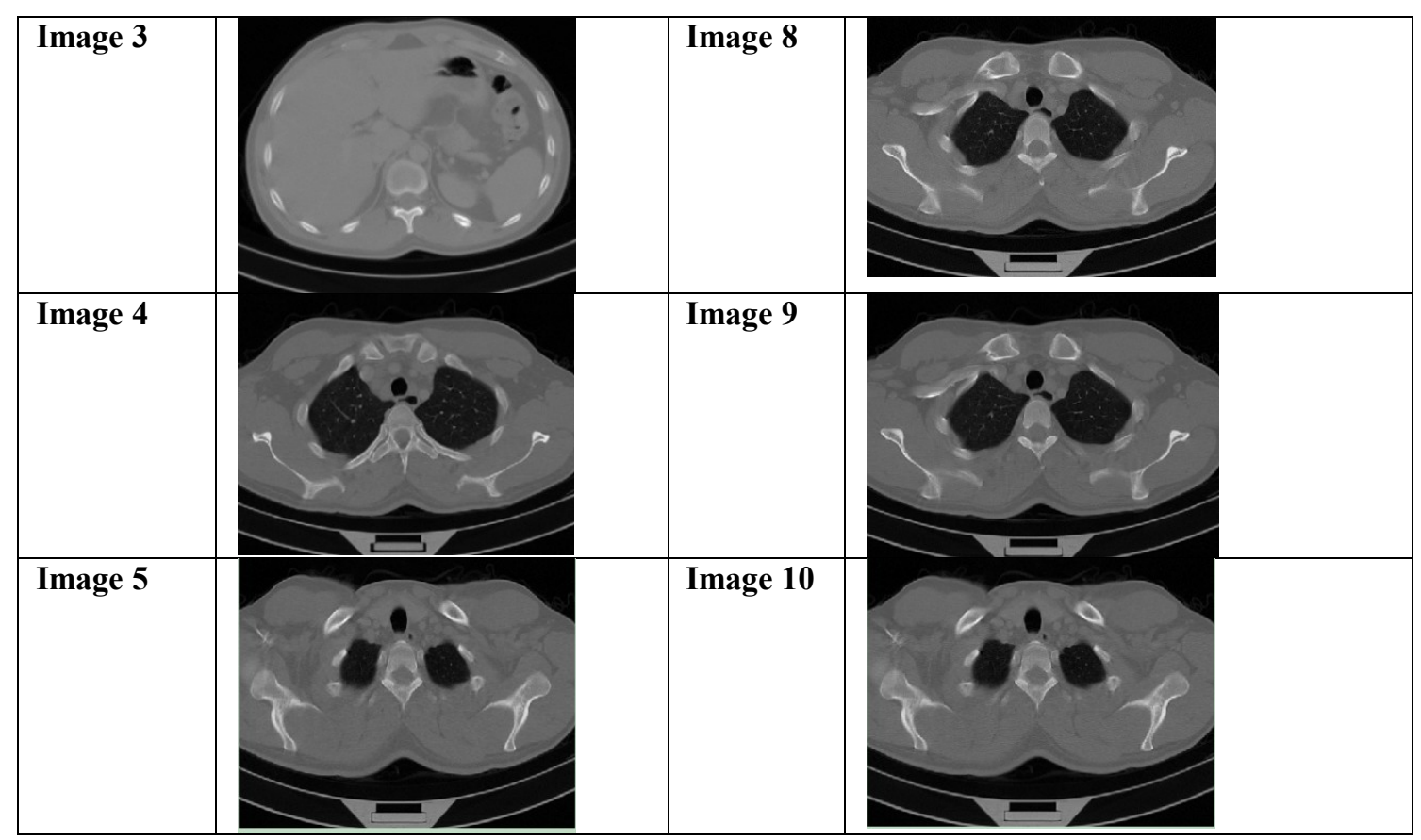

Table 1

1.2 Various Parameters values corresponding to 10 images:

\begin{tabular}{|c|c|c|c|c|c|c|c|c|c|c|}
\hline \multirow{2}{*}{$\begin{array}{l}\text { PARAMIETER } \\
\text { NAMIE }\end{array}$} & \multicolumn{10}{|l|}{ VAIUE } \\
\hline & IMAGE 1 & IMLAGE 2 & IMLAGE 3 & IMAGE 4 & IMAGE 5 & IMLAGE 6 & IMLAGE7 & IMAGE 8 & IMAGE9 & IMAGE10 \\
\hline FALSE POSITTVE & 8.5654 & 8.6661 & 13.5812 & 5.9042 & 1.1536 & 0.54845 & 6.5275 & 7.3078 & 5.8391 & 0.30633 \\
\hline F MEASURE & 1.3524 & 1.38889 & 0.99337 & 1.5294 & 1.5653 & 1.8835 & 1.3822 & 1.4439 & 1.4983 & 1.8168 \\
\hline SENSTIVTTY & 11.1109 & 27.5201 & 27.5201 & 11.6299 & 9.9205 & 28.6037 & 25.0802 & 16.5573 & 18.1936 & 23.1929 \\
\hline PRECISION & 0.72001 & 0.71243 & 0.58314 & 0.81854 & 0.8497 & 0.97381 & 0.71066 & 0.75487 & 0.78134 & 0.94542 \\
\hline RECALL & 1.1109 & 12.0502 & 3.3504 & 11.6299 & 9.9205 & 28.6037 & 25.0802 & 16.5573 & 18.1936 & 23.1929 \\
\hline GMEAN & 16.7486 & 11.5497 & 9.5346 & 17.6782 & 13.8314 & 21.5439 & 13.8166 & 14.1627 & 21.4934 & 25.4649 \\
\hline ACCURACY & 83.423 & 81.5635 & 83.3277 & 82.7767 & 81.7404 & 84.9734 & 83.4425 & 82.0116 & 80.1946 & 84.6884 \\
\hline EFFICIENCY & 98.4077 & 98.8649 & 96.4205 & 96.4628 & 121.324 & 108.0595 & 106.4398 & 99.1888 & 102.0485 & 123.3871 \\
\hline
\end{tabular}

Table 2

1.3 ROC Curve corresponding to 10 Images

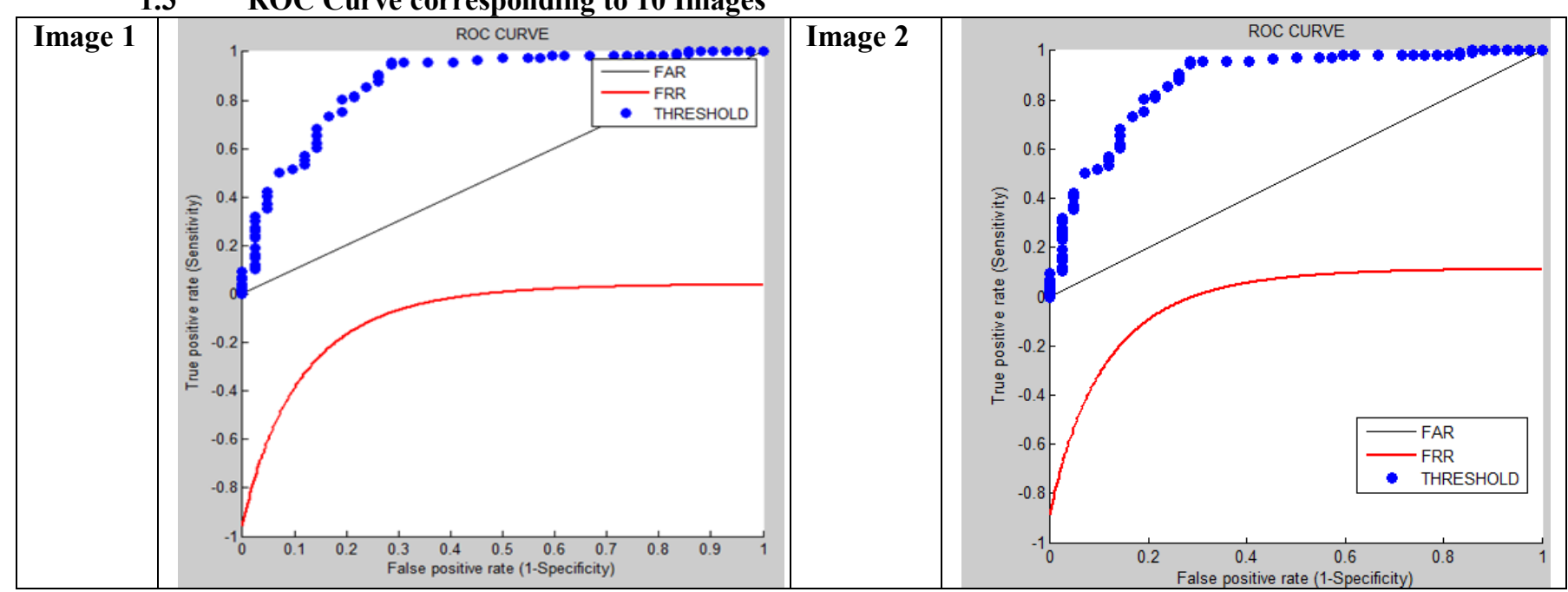


An Intelligence System for Detection of Cancer and Diagnosis

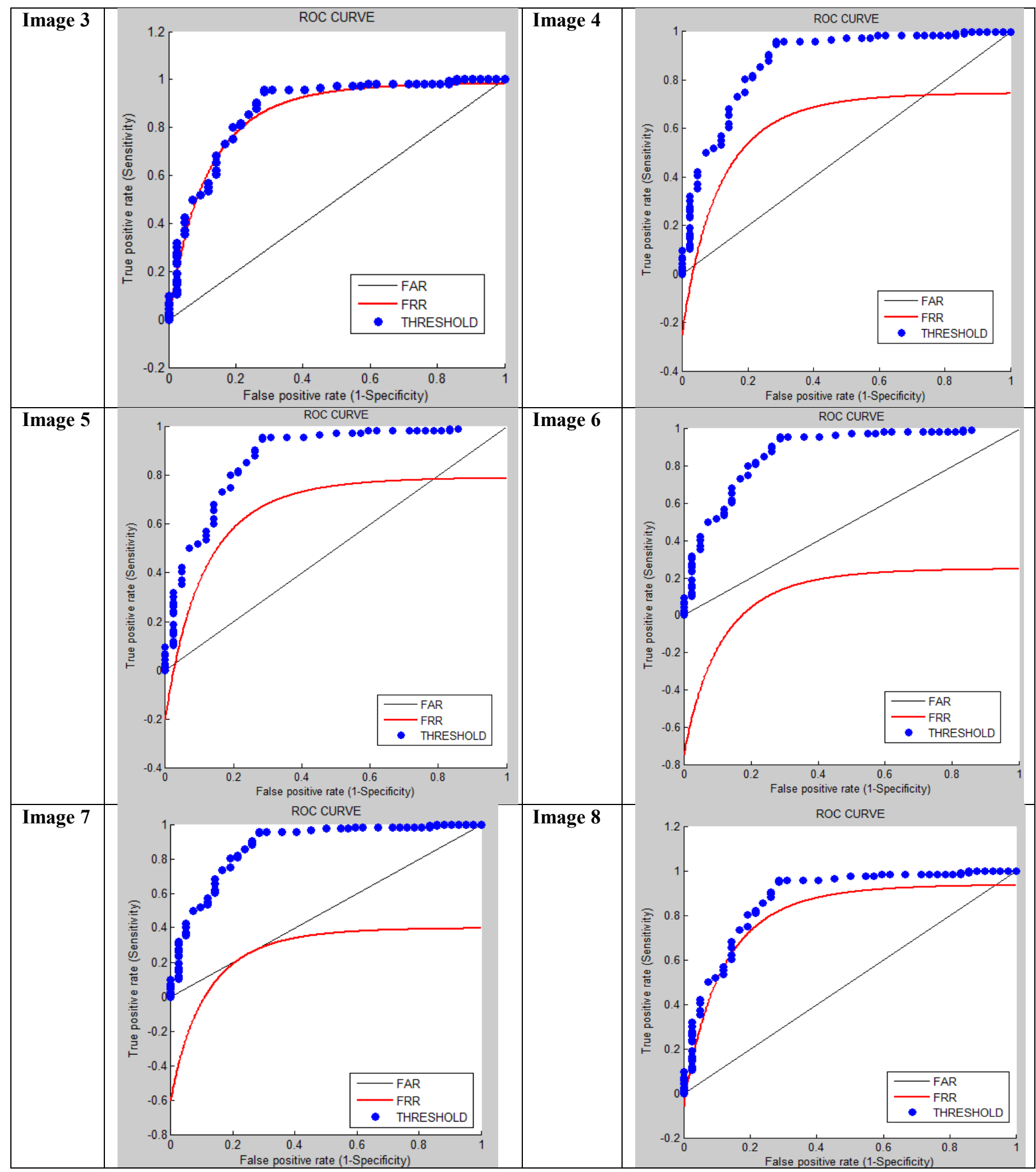




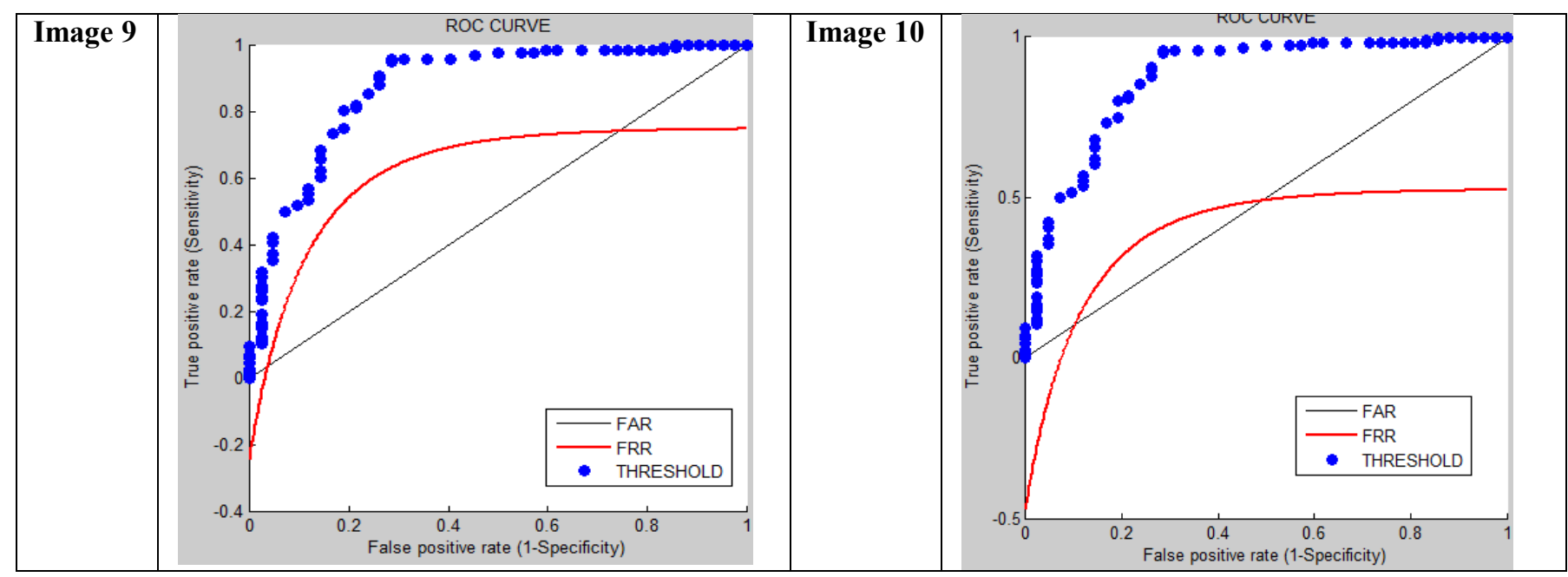

Table 3

\section{Conclusion}

This paper has focused on the CBIR application in diagnosis cancer disease. The overall efficiency of MRI brain image retrieval can be improved by the usage of appropriate feature vector. Nevertheless, certain efforts within the engineering community are worth nothing. Content- based image retrieval of diagnosis of cancer disease has achieved a degree of maturity, albeit at research level at a time of significant. However, the field has get to make considerable attacks into mainstream clinical practice, medical research or training.

A technique that effectively uses most of the information from image is backbone of an efficient content-based image retrieval system for medical database. In this paper we have developed a CBIR system based on feed forward back propagation algorithm techniques for future extraction and similarity measurements. It has been observed from the experimental that feed-forward back propagation neural network gives better results in terms of efficiency, precision and recall.

\section{REFERENCES}

[1] Ms. Sonali B. Maind, Ms. Priyanka Wankas, Research Paper on Basic of Artificial Neural Network, International Journal on Recent and Innovation Trends in Computing and Communication, Volume 2, Issue 1, pp. 96-100.

[2] Vinicias Goncalves Maltarollo, Kathia Maria Honorio and Alberico Borges Ferreira da Silva, Application of Artificial Neural Network in Chemical Problems, INTECH, Link: http://dx.doi.org/10.5772/51275

[3] http://en.wikipedia.org/wiki/Adaptive learning

[4] http://en.wikipedia.org/wiki/Content-based image retrieval

[5] H. Chuctaya, et al., "M-CBIR: A Medical Content Based Image Retrieval System Using Metric Data Structures", Computer Science Society (SCCC), $30^{\text {th }}$ IEEE International Conference of the Chilean, 2011.

[6] L. Rodney Long, Sammer Antani, Thomas M. Deserno, George R. Thoma, Content Based Image Retrieval in Medicine, Int J Healthe Inf Syst Inform. Jan 1, 2009; 4(1): 1-16.

[7] J.C. Caicedo, F.A. Gonzalez, E. Romero, "Content Based Medical Image Retrieval Using Low Level Visual Features and Modality Identification", Advances in Multilingual and Multimodal Information Retrieval, Springer, pp. 615-622, 2008.

[8] B.C. Ramamurthy, K.R. Chandran, S. Aishwarya, P.Janaranjani, "CBMIR: Content Based Image Retrieval using Invariant Moments, GLCM and Grayscale Resolution for Medical Images”, European Journal of Scientific Research, Vol. 59, Issue 4, p. 460, 2011.

[9] Sh. Akbarpour, A Review On Content Based Image Retrieval Inmedical Diagnosis, International Journal on Technical and Physical Problems of Engineering, Issue 10, Vol. 5, pp. 148-153.

[10] http://en.wikipedia.org/wiki/Backpropagation 